\title{
Lorsque la marque du conditionnel est une particule mobile : le cas du russe
}

\author{
Christine Bonnot*, Tatiana Bottineau**
}

\section{LE CONDITIONNEL RUSSE : PROBLEMES DE DEFINITION}

En russe, le conditionnel ${ }^{1}$ n'est pas un tiroir verbal, mais a pour marque une particule enclitique, by, dont nous nous proposons de montrer qu'elle porte non sur le verbe, mais sur la proposition entière. Cette situation originale pour une langue hautement flexionnelle est le fruit d'une profonde transformation du système des conjugaisons du vieux russe.

En vieux russe, le conditionnel était une forme verbale analytique composée de l'aoriste de l'auxiliaire "être » byti et d'un participe parfait actif en -1: byx" privel" "j'amènerais/j'aurais amené"2. Il entrait dans le même paradigme que trois autres formes de structure semblable : parfait (Etre-PRESENT + participe en -1 ), plus-que-parfait (Être-PARFAIT ${ }^{3}+$ participe en -1 ) et futur antérieur (ÊtreFUTUR + participe en -1). Ce système a été démantelé en russe moderne. Le parfait est devenu un temps simple, du fait de la disparition des formes personnelles du verbe «être » au présent. Réduit à la seule forme en -l qui s'accorde en genre et nombre, il constitue aujourd'hui l'unique passé du russe, les anciens temps simples, imparfait et aoriste, étant parallèlement tombés en désuétude. Le futur antérieur a disparu : le russe n'a plus de temps relatif et ne marque pas plus l'antériorité dans le futur que l'antériorité ou l'ultériorité dans le passé. Au plus-que-parfait et au conditionnel, enfin, les formes de parfait et d'aoriste de l'auxiliaire sont devenues invariables et se sont grammaticalisées en particules enclitiques modales, bylo et by, qui ont pris une certaine autonomie par rapport au verbe : elles ne sont plus exclusivement corrélées à l'ancien participe en -1, devenue forme de passé, et sont mobiles au sein de l'énoncé ${ }^{4}$.

Ainsi by entre aujourd'hui en combinaison régulière :

- avec le passé (ancien participe en -1) s'accordant avec le sujet au nominatif :

* INaLCO (Paris) \& CNRS UMR 8202 : SeDyL/CELBS. Courriel : BonnotCh1@ aol.com ** INaLCO (Paris) \& CNRS UMR 8202 : SeDyL/CELBS. Cour. : tbottineau @ gmail.com ${ }^{1}$ «Conditionnel » est le terme consacré chez les slavistes français pour désigner un mode aux emplois très divers que les grammairiens russes appellent généralement « subjonctif » (soslagatel'noe naklonenie), ou parfois « hypothétique » (predpoložitel'noe naklonenie). ${ }^{2}$ La forme d'aoriste de l'auxiliaire n'était pas première, mais avait remplacé une ancienne forme d'optatif avec laquelle elle est encore en concurrence dans les textes vieux slave.

${ }^{3}$ A l'origine, l'auxiliaire était à l'imparfait, qui a ensuite été remplacé par le parfait.

${ }^{4}$ Sur bylo, cf. Bottineau, 2009. 
(1) Bez raboty on by ne vyderžal i dvux dnej. Sans travail il-Nom by NEG tenir-PSE même deux jours Sans travail, il ne tiendrait même pas deux jours.

- avec un infinitif ; le sujet, lorsqu'il est exprimé, est alors au datif :

(2) Bez raboty emu by ne vyderžat' i dvux dnej. Sans travail il- DAT by NEG tenir-INF même deux jours Sans travail, il ne pourrait même pas tenir deux jours.

- avec un prédicat nominal :

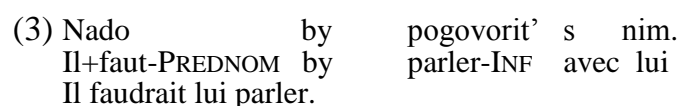

De façon plus occasionnelle by peut également apparaître avec l'impératif :

(4) Proživi by Lenin eščë paru let... (A. Terexov, Babaev) ${ }^{5}$ Vivre-IMPÉR by Lénine-Nom encore paire d'années Si Lénine avait vécu encore deux ans...

ou sans aucune forme verbale :

\section{(5) Skoree by leto! Plus+tôt by été Vivement l'été !}

Cette évolution radicale entraîne des divergences dans la définition même du mode conditionnel en russe moderne. La plupart des chercheurs russes opposent la combinaison [by+passé], directement héritée du vieux-russe, dans laquelle ils voient une forme analytique appartenant au paradigme du verbe, aux autres emplois de by, considérés comme des combinaisons syntaxiques à valeur modale. Cette conception, qui rejette tout parallélisme entre des énoncés pourtant aussi proches que (1) et (2), explique qu'il n'existe pas en Russie, à notre connaissance, d'étude systématique de l'ensemble des emplois de by. Ils ne sont abordés que de façon parcellaire dans des travaux d'inspiration onomasiologique énumérant les procédés susceptibles d'exprimer telle ou telle valeur modale (hypothèse, souhait, conseil, etc.) ${ }^{6}$.

\footnotetext{
${ }^{5}$ La plupart de nos exemples sont empruntés au Corpus National de la Langue Russe (www.ruscorpora.ru), dont nous remercions les auteurs.

${ }^{6}$ Outre le poids de la diachronie, ce refus de mettre la combinaison avec le passé sur le même plan que les autres nous semble dû à ce qu'elle est la seule à contenir une indication temporelle apparemment contradictoire avec l'interprétation du conditionnel, qui en russe peut renvoyer indifféremment à une situation passée, présente ou future. Cette difficulté disparait si l'on considère que la valeur de passé associée au suffixe -1 ne lui est pas intrinsèque, mais n'est qu'une valeur dérivée par défaut d'une opération plus
} 
La plupart des slavistes français partagent la conception inverse défendue par P. Garde qui, dans sa thèse magistrale de 1963, appelle «conditionnel » «tout prédicat contenant la particule by $»(1963$ : 8). Adoptant une démarche strictement sémasiologique et structuraliste, P. Garde met en évidence les facteurs intervenant dans la répartition entre conditionnels « personnel» (by + Passé), «impersonnel » (by + Infinitif) et «nominal » (by dans une phrase sans verbe) et montre que chacun de ces types peut donner naissance, en fonction du contexte, à deux grands groupes de valeurs modales : «intervention » et «fait conditionné ». Sa monographie reste à ce jour la description la plus claire et la plus complète des emplois de by dans les indépendantes comme dans les subordonnées et c'est d'elle que nous sommes parties pour notre recherche.

Nous nous en démarquons cependant sur un point important : bien que by conditionne la forme du prédicat (il ne peut apparaître avec le présent de l'indicatif), nous pensons qu'il n'appartient pas à celui-ci, mais porte sur l'ensemble de la proposition ${ }^{7}$, comme le montre sa mobilité au sein de celleci. Comme toutes les autres particules enclitiques du russe, qu'elles soient ou non d'origine verbale, il admet en effet deux positions :

1 position de Wackernagel, après le premier mot accentogène soit de l'énoncé pris globalement, soit de sa seule partie rhématique; dans les subordonnées, après la conjonction, avec laquelle il y a parfois fusion (čto «que » + by $\rightarrow$ čtoby) ;

2 position focale : après un constituant foyer de contraste (souvent le prédicat, mais pas nécessairement).

Ces deux positions peuvent éventuellement se cumuler, comme dans les énoncés exprimant la crainte, où une inversion obligatoire de l'ordre canonique rejette à l'initiale le prédicat focalisé : Ne opozdal by on! (NEG être+en+retardPSE by il-NOM) « Pourvu qu'il ne soit pas en retard ! ».

Ces variations positionnelles n'ont pratiquement jamais été étudiées et sont généralement considérées comme libres ou mises au compte de la prosodie, alors que nous avons pu constater qu'elles peuvent modifier l'interprétation de la particule. Ce sera l'objet de notre troisième section.

\section{LES VALEURS DU CONDITIONNEL RUSSE}

Pouvant renvoyer indifféremment à une situation postérieure, antérieure ou concomitante au moment de référence, le conditionnel russe a un spectre de valeurs très large, qui recouvre aussi celles du subjonctif des langues romanes, d'où le flottement terminologique signalé dans la note 1. Cependant, il n'exprime qu'une partie des valeurs du conditionnel français. En particulier, il ne

abstraite de « distanciation » qui peut se faire aussi bien sur le plan temporel que sur le plan modal. Cf. l'imparfait français, a priori perçu comme renvoyant au passé, sauf s'il est précédé de «si » («Ah! Si j'étais riche! »).

${ }^{7}$ Point de vue déjà exprimé par B. Unbegaun (1951 : 251), qui ne l'a pas développé. 
sert jamais à marquer l'ultériorité dans le passé ${ }^{8}$, et ne peut pas non plus exprimer une conjecture ou un propos rapporté sous réserve (« emprunt »).

Cette différence s'explique par l'étymologie. Combinant une marque d'imparfait à une marque d'infinitif, le conditionnel français présente le procès comme validable à partir d'un repère translaté fourni par le contexte (repère temporel antérieur au moment de référence, énonciateur rapporté ou état de choses fictif introduit par une protase à l'imparfait). Issue d'une forme d'aoriste, la particule russe by ne suppose pas de translation, mais marque une rupture directe avec la situation de référence Sit : l'état de choses $\mathrm{P}$ exprimé dans la proposition où apparaît by est opposé à un état de choses $\mathrm{P}^{\prime}$ actualisé ou susceptible d'être actualisé en Sit. Suivant la nature des repérages énonciatifs, cette opposition donne naissance à deux grands groupes de valeurs :

- contrefactuel : construction d'une relation d'inférence à partir d'un état de choses $\mathrm{P}$ opposé à $\mathrm{P}$ ' validé en Sit. $\mathrm{Cf}$. (6), où by est déjà présent dans la protase puisqu'on ne construit pas de repère translaté :

(6) Esli by ne derev'ja, rečka vysoxla by. Si by NEG arbres-NOM rivière-NOM s'assécher-PSE by S'il n'y avait pas les arbres, la rivière se serait asséchée/s'assècherait.

- conseil/souhait : construction d'un état de choses $\mathrm{P}$ à la fois présenté comme souhaitable et opposé à $\mathrm{P}$ ' effectivement validé ou validable ${ }^{9}$. Cf. (7), qui suggère à l'interlocuteur une action qu'il n'a pas pensé à faire de lui-même, alors qu'elle est appelée par la situation, et (8), qui exprime un souhait que le locuteur sait par avance irréalisable :

(7) Zdes' dušno. Ty by provetril nemnogo.

Tu-NOM by aérer-PSE un+peu

On étouffe ici. Tu devrais/pourrais aérer un peu.

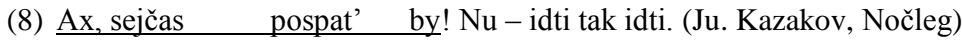

\footnotetext{
${ }^{8}$ La concordance des temps est limitée en russe : absente dans le discours indirect, elle ne concerne que l'expression de la concomitance dans les autres contextes. La postériorité par rapport à un repère s'exprime toujours de la même façon (futur imperfectif ou présent perfectif) que ce repère soit présent, passé ou futur.

${ }^{9}$ Cette caractérisation s'oppose à celle que propose P. Garde qui, regroupant ces valeurs sous le terme de «conditionnel d'intervention", considère qu'elles apparaissent en « contexte zéro », c'est-à-dire en l'absence de tout élément susceptible d'infléchir la valeur du conditionnel, la valeur de contrefactuel supposant elle la présence d'un élément exprimant une hypothèse. Or l'examen du corpus montre que contrairement à l'impératif, qui est susceptible d'exprimer une injonction ex nihilo, le conditionnel d'intervention souligne toujours une contradiction entre ce qui est souhaitable et ce qui est validé ou validable dans la situation de référence. C'est la raison pour laquelle un «conseil» exprimé au conditionnel est perçu comme moins catégorique qu'une «injonction » exprimée à l'impératif: le procès que l'interlocuteur est invité à valider ne relève pas du seul arbitraire du locuteur, mais est présenté comme destiné à lever cette contradiction.
} 
Ah, maintenant dormir-INF by !

Ah ! Là, je piquerais bien un petit somme ! Mais puisqu'il faut y aller...

L'interprétation modale exacte dépend non seulement des déterminations contextuelles et situationnelles, mais aussi de la forme et de l'aspect du prédicat, de la personne du sujet et de la place de by. C'est ce dernier paramètre, généralement ignoré, que nous nous proposons d'examiner maintenant. Notre hypothèse est que, en devenant une particule enclitique, la marque du conditionnel by a acquis les propriétés catégorielles de cette classe de marqueurs discursifs et que sa place est régie par les mêmes règles générales que celles que nous avons déjà mises en évidence pour d'autres particules enclitiques comme že (Bonnot, 2001-2002) ou bylo (Bottineau, 2009). Comme toutes les autres particules, by a pour fonction de spécifier le segment qui constitue sa portée (la proposition exprimant l'état de choses $\mathrm{P}$, présenté comme en rupture avec Sit) en l'opposant à une valeur concurrente (un état de choses $\mathrm{P}$ ' attesté en Sit). Sa position au sein de sa portée dépend du statut respectif des valeurs concurrentes $\mathrm{P}$ et $\mathrm{P}$ ' : lorsque $\mathrm{P}$ est introduit en opposition à $\mathrm{P}$ ' seul existant au départ, il est en position de Wackernagel, et lorsque $P$ est sélectionné au sein d'un paradigme $\mathrm{P} / \mathrm{P}$ ' préexistant, il est en position focale, après le mot sur lequel se joue l'opposition entre les deux valeurs.

Pour montrer comment les variations positionnelles de by, tout en obéissant à ce mécanisme très général, modifient l'interprétation modale, nous prendrons l'exemple des systèmes hypothétiques formés avec une proposition conditionnante introduite par la conjonction esli «si ». Nous examinerons la place de by au sein de cette conditionnante, sans envisager, faute de place, ses variations au sein de la principale, qui obéissent aux mêmes règles.

\section{UN EXEMPLE DES VARIATIONS POSITIONNELLES DE BY : LES SUBORDONNEES CONDITIONNANTES INTRODUITES PAR ESLI "SI"}

\subsection{Position de Wackernagel : by suit immédiatement la conjonction}

C'est le cas de très loin le plus fréquent, il correspond à une intonation neutre, sans mise en relief contrastive, et est le seul qui soit enseigné aux étrangers :

$$
\begin{aligned}
& \text { (9) - Mama, esli by ja golosoval, to postavil by v každom } \\
& \text { si by je-Nom voter-Pse, alors mettre-PSE by dans chaque } \\
& \text { kružočke pečat', čtoby nikto ne obižalsja. A teper' odin budet radostnyj, a } \\
& \text { case tampon } \\
& \text { ostal'nye budut grustit'. (Internet) } \\
& \text { Maman, si je pouvais voter, je tamponnerais chaque case pour que personne } \\
& \text { ne soit vexé. Parce que là, il y en a un qui sera content, et les autres qui seront } \\
& \text { tristes. }
\end{aligned}
$$

La conjonction esli, issue de la fusion du présent du verbe «être » avec la particule formant des interrogations fermées li $(<*[$ est' + li] : litt. « est ou non ») présente l'état de choses $\mathrm{P}$ dénoté dans la proposition qu'elle introduit comme 
appartenant à une alternative $\mathrm{P} / \mathrm{P}$ '. Lorsqu'elle est suivie de l'indicatif, les deux termes de l'alternative apparaissent également validables. Ainsi, l'indicatif passé en contexte itératif signifierait la validation tantôt de P, tantôt de P' : Esli ja golosoval, to za levyx ( $\mathrm{Si}$ je voter-PSE, alors pour gauche) «Si je votais, c'était pour la gauche »), tandis que l'indicatif futur renverrait à une situation incertaine, le locuteur n'ayant pas encore choisi s'il validerait P ou P' : Esli ja budu golosovat', to za levyx (Si je être-FUT voter-INF, alors gauche) «Si je vote, ce sera pour la gauche ».

L'introduction de by, qui spécifie $\mathrm{P}$ comme étant en rupture avec la situation de référence Sit, rompt l'équilibre entre les deux termes de l'alternative. P est présenté comme relevant de la pure fiction, $\mathrm{P}$ ' étant seul attesté ou attestable : chacun sait que les enfants ne votent pas. Dans la mesure où il s'agit d'un fait acquis que nul ne songe à remettre en question, by ne peut être qu'en position de Wackernagel, conformément à notre hypothèse : $\mathrm{P}$ est second par rapport à P', seul existant au départ.

Le placement de la particule en position focale rééquilibre partiellement les deux termes de l'alternative sans pour autant faire disparaître l'interprétation contrefactuelle. Conformément à notre hypothèse, il signifie en effet que $\mathrm{P}$ est sélectionné au sein d'un paradigme préexistant de deux valeurs concurrentes dont chacune a un support subjectif. Le mode de construction de ces deux valeurs n'est pas le même suivant que by est placé après le verbe ou après le sujet.

\subsection{Position focale après le verbe : confrontation de points de vue.}

Le placement de by après le verbe indique que $\mathrm{P}$ est sélectionné au sein d'un paradigme de deux propositions concurrentes $\mathrm{P}$ et $\mathrm{P}$ ' qui ne diffèrent que par la modalité affirmative/négative dont le verbe est porteur. La référence à cette concurrence première peut renvoyer à l'existence de deux points de vue contraires sur leurs valeurs de vérité respectives :

(10) (Le locuteur conteste l'idée selon laquelle le film russe « les Douze » n'aurait pas obtenu l'Oscar du meilleur film étranger pour des raisons politiques.)

- Ja ne soglasen s ètoj postanovkoj voprosa. V kategorii «Lučšij fil'm na inostrannom jazyke » sošlis' dva titana - Mixalkov s «12» i Vajda s

« Katyn'ju ». Čtoby ne obižat' ni togo, ni drugogo, pobedu prisudili tret'emu - avstrisjskomu režissëru za «Fal'šivomonetčikov ».

Esli že amerikancy dejstvitel'no xoteli by podpustit'

Si par+contre Américains-Nom réellement vouloir-PSE by laisser+entrer $\mathrm{v}$ ètu istoriju politiki, to nagradu otdali by «Katyni ». dans cette histoire de+la+politique, alors prix donner-PSE by «Katyn-DAT » - Je ne suis pas d'accord avec cette façon de poser la question. Dans la catégorie du «Meilleur film en langue étrangère », deux géants étaient en concurrence, Mikhalkov avec « les Douze » et Wajda avec « Katyn ». Pour ne vexer ni l'un, ni l'autre, on a donné la victoire à un troisième, un metteur en scène autrichien, pour «les Faussaires ». Alors que si les Américains avaient vraiment voulu mêler la politique à cette affaire, ils auraient attribué l'Oscar à «Katyn ». (Argumenty i fakty) 
Après avoir défendu son propre point de vue P' en expliquant ce qui avait selon lui motivé le choix du jury, le locuteur reconsidère le bien-fondé de l'hypothèse $\mathrm{P}$ défendue par son interlocuteur en envisageant sa validation fictive, ce qui lui permet de la réfuter par l'absurde : $\mathrm{P}$ validé aurait abouti à un état de choses autre que celui que l'on peut objectivement constater. On note que cette remise en concurrence de $\mathrm{P}$ et $\mathrm{P}$ ' entraîne la présence d'un accent de contraste sur le marqueur discursif dejsvitel'no ( « réellement»). Cet accent et la particule adversative že («par contre») disparaitraient avec by en position de Wackernagel :

(10a) Esli by amerikancy dejstvitel'no xoteli podpustit' $\mathrm{v}$ ètu si by Américains-NOM réellement vouloir-PSE laisser-entrer dans cette

istoriju politiki, to nagradu otdali by «Katyni ».

histoire de-la-politique, alors prix attribuer-PSE by «Katyn »-DAT $\mathrm{Si}$ les Américains avaient réellement voulu mêler la politique à cette affaire, ils auraient attribué l'Oscar à « Katyn ».

La stratégie aurait été un peu différente : s'inscrivant dans la continuité de ce qui précède, $\mathrm{P}$ aurait d'emblée été présenté comme erroné, alors que dans le texte d'origine, le locuteur, abandonnant momentanément sa propre position, feint d'envisager la possibilité de valider $\mathrm{P}$ pour mieux l'écarter.

Si le maintien de la concurrence entre $P$ et $P$ ' est rhétorique en (10), ailleurs il peut traduire le doute du locuteur, qui n'est pas sûr d'avoir raison en validant $\mathrm{P}^{\prime}$ :

(11) (Une fillette refuse de croire son amie qui lui raconte avoir chez elle de petites créatures invisibles qui lui ont sauvé la vie.)

- I vovse ty obmanščica, - [...]. - I nikakix nevidimok u tebja net. I nikto tebja ne spasal. Esli byli by, tak pokazala b...

si être-PSE-PL by, alors montrer-PSE-FEM b $(y))^{10}$

- Nu pojdëm, pojdëm, uvidiš’ sama.

- A oni maljusen'kie?

Devočki pomčalis' k pod"ezdu. (M. Sergeev, Volšebnaja galoša)

- Et tu n'es qu'une menteuse. [...] Tu n'as aucune créature invisible chez toi. Et personne ne t'a sauvée. $\mathrm{Si}$ jamais tu en avais, tu pourrais les montrer...

- Eh bien, allons-y, allons-y, tu verras toi-même.

- Et elles sont toutes petites?

Les deux fillettes coururent vers l'entrée de l'immeuble.

Le placement de by après le verbe, qui entraîne un accent de contraste sur celui-ci, traduit une hésitation confirmée par les points de suspension: si la fillette considère a priori que $\mathrm{P}$ relève du faux, elle reste prête à l'admettre pour vrai si on lui en fournit la preuve. L'argument apparent en faveur de P' se double en fait d'une invitation implicite à prouver $\mathrm{P}$, comme le montre l'empressement avec lequel elle accepte d'aller voir les créatures invisibles, oubliant aussitôt tous

${ }^{10} \mathrm{~b}$ est un allomorphe de by pouvant apparaître après voyelle dans la langue familière. 
les doutes qu'elle avait sur leur existence (cf. A oni maljusen'kie? «Et elles sont toutes petites ?»). Le placement de by en position de Wackernagel, excluant d'emblée toute possibilité de valider $P$, rendrait l'énoncé beaucoup plus catégorique et serait incompatible avec un tel revirement dans le contexte droit :

(11a) Esli by byli, tak ty by by
si by être-PSE-PL, alors tu-Nom by montrer-PSE $\mathrm{Si}$ tu en avais, tu les montrerais.

Les deux verbes sont ici porteurs d'accents neutres et l'intonation ne laisse pas de place au doute, comme le symbolise le point final ${ }^{11}$.

\subsection{Position focale après le sujet : entre contrefactuel et potentiel.}

Comme dans le cas précédent, la position focale implique la préconstruction d'un paradigme où les deux valeurs $\mathrm{P}$ et $\mathrm{P}$ ' sont en concurrence, ce qui s'interprète comme une réouverture de l'alternative : bien que la présence de by signifie a priori la validation de P', P n'est pas définitivement écarté. La différence est que la sélection de l'une ou l'autre valeur ne relève plus de l'appréciation portée par l'énonciateur sur un état de choses dont il n'a pas une connaissance directe (modalité épistémique), mais de la responsabilité du sujet du procès, dont le comportement actuel pourrait éventuellement changer : l'interprétation oscille entre contrefactuel et potentiel.

(12) (Un kolkhozien de 70 ans explique pourquoi il ne peut pas partir à la retraite.) - Ja znaju, kto na moë mesto lezet. On za mesjac razob'ët traktor i vsju svarku pogubit. Kak togda brigada budet rabotat'? [...] Oni že vsjakij den' kuvyrkajutsja, texniku b’jut, ja ix činju. A esli ja by kuvyrkalsja vmeste

$$
\text { s nimi? Kto by varil? }
$$

Et si je by s'amuser-PSE_ensemble avec eux ? Qui by souder-PSE?

zakuvyrkaetsja.Vot vsë i končitsja. Tak čto nado rabotat'.

Comment dans ces conditions la brigade pourra-t-elle travailler? [...] Ils passent leur temps à s'amuser, ils cassent les outils, moi je les répare. Et si je me mettais moi aussi à faire la fête avec eux ? Qui ferait les soudures? Mon successeur, lui, ira faire la fête dès le premier jour. Et ce sera la fin. Je suis donc obligé de continuer. (B. Ekimov, Na xutore)

Porteur d'un accent de contraste, le sujet est opposé à la fois aux autres kolkhoziens évoqués dans le contexte gauche, et à son successeur éventuel, évoqué dans le contexte droit. Le placement de la particule en position focale permet à l'énoncé de fonctionner comme un pivot entre ces deux oppositions qui appartiennent à des plans temporels et modaux différents. L'opposition avec les

${ }^{11}$ On note que by quitte également la position focale dans la principale, puisque l'état de choses $\mathrm{Q}$ dénoté par celle-ci est totalement écarté par l'exclusion définitive de $\mathrm{P}$, alors qu'en (11), qui était une invite déguisée, il constituait une faible éventualité. 
kolkhoziens relève de l'acquis et confère à $\mathrm{P}$ une valeur contrefactuelle : le sujet, contrairement à ses collègues, ne fait pas la fête au travail. L'opposition avec son successeur éventuel implique, elle, une projection dans l'avenir, ce qui permet de réintroduire $\mathrm{P}$ comme une éventualité que le sujet reste toujours libre de valider, même s'il se refuse a priori à le faire. En invitant son interlocuteur à imaginer les conséquences néfastes qu'entraînerait un tel choix, le locuteur fait comprendre pourquoi il ne peut pas laisser son poste à quelqu'un qui, placé devant la même alternative, s'empresserait, lui, de faire la fête avec les kolkhoziens.

Le placement de by en position de Wackernagel ferait perdre à l'énoncé cette dimension rhétorique en limitant sa pertinence au seul plan du présent, où $\mathrm{P}$ n'est pas en concurrence avec P' :

(12a) Esli by ja kuvyrkalsja vmeste $\mathrm{s}$ nimi, to kto by varil?

Si by je s'amuser-PSE ensemble avec eux, alors qui by souder-PSE Si je faisais la fête avec eux, qui ferait les soudures?

L'énoncé ne ferait que commenter le caractère peu satisfaisant de la situation existante, sans anticiper sur une possible aggravation dans l'avenir. Le caractère purement fictif de $\mathrm{P}$ interdirait de l'énoncer sous la forme d'une question invitant l'interlocuteur à imaginer les conséquences qu'entraînerait sa validation, et obligerait à articuler protase et apodose dans un même énoncé complexe. Enfin, bien que toujours opposé aux kolkhoziens, le sujet perdrait son accent de contraste: nous en concluons qu'en (12) l'opposition marquée par celui-ci n'était pas syntagmatique, mais paradigmatique, entre deux avatars du sujet, celui qui a toujours validé $\mathrm{P}^{\prime}$, et celui qui pourrait choisir de valider $\mathrm{P}$.

Cette interprétation est confirmée par l'exemple suivant, où le sujet suivi de by est également porteur d'un accent de contraste, bien qu'il n'entre dans aucune opposition syntagmatique :

(13) ...ot Ritinoj materi, dovol'no neuravnovešennoj damočki, kotoruju my odnaždy posetili na Kèjp Kode i kotoraja s tex por, v častyx pis'max [...] vsë govorila mne, kak udivitel'no eë doč' i ja podxodim drug družke i kak čudno bylo by, esli my by ženilis'

comme merveilleux être-PSE by, si nous by se-marier-PSE

...de la mère de Rita, une petite dame plutôt exaltée que nous étions allés voir une fois à Cape Cod et qui depuis, dans des lettres fréquentes [...] ne cessait de me répéter à quel point sa fille et moi allions bien ensemble et comme ce serait merveilleux si nous nous décidions à nous marier. (V.Nabokov, Lolita)

Le placement de by après le sujet s'interprète comme une pression active du locuteur sur son interlocuteur. Bien que la mère sache très bien que le narrateur et sa fille n'ont pas l'intention de se marier, elle garde l'espoir qu'ils changeront d'avis : la présence de by prend acte de la validation actuelle de P', et sa position focale souligne que $\mathrm{P}$ reste validable pour peu que les sujets le décident. Si la particule était en position de Wackernagel, la mère n'exprimerait qu'un souhait purement théorique : bien que considérant que le mariage de sa fille avec le narrateur serait une bonne chose, elle serait résignée à ce qu'il n'ait jamais lieu. 


\section{CONCLUSION}

La grammaticalisation de la forme d'aoriste de l'auxiliaire «être » en particule clitique a profondément modifié le statut du conditionnel russe, qui n'est plus une catégorie verbale, mais un trait attribué à l'ensemble de la proposition. Désormais compatible avec différentes formes verbales (autres que le présent de l'indicatif) et pouvant aussi apparaître en l'absence de tout verbe, la particule by a le même fonctionnement syntaxique que les autres particules clitiques à sémantique modale (bylo, issu du parfait du même auxiliaire) ou argumentative (že d' «évidence »). Présentant l'état de choses $\mathrm{P}$ exprimé par la proposition où elle apparaît comme construite en rupture avec la situation de référence, elle l'oppose à un état de choses $\mathrm{P}$ ' qui est lui, actualisé ou susceptible de l'être. Loin d'être aléatoires, ses variations positionnelles participent au calcul de l'interprétation modale, comme l'a montré l'étude des propositions conditionnantes introduites par la conjonction esli: le placement après la conjonction (position de Wackernagel) signifie que P' est premier et seul attesté (contrefactuel pur); le placement après le verbe réintroduit la possibilité de valider $\mathrm{P}$, soit pour mieux l'exclure (rhétorique argumentative), soit parce que le locuteur a des doutes sur la valeur qu'il a sélectionnée (dédoublement); le placement après le sujet souligne que la validation de $\mathrm{P}$ ou $\mathrm{P}$ ' est du ressort de celui-ci et qu'il reste libre de valider $\mathrm{P}$, même s'il valide actuellement $\mathrm{P}$ ' (nuance de potentiel).

\section{REFERENCES BIBLIOGRAPHIQUES}

Bonnot Ch., 2001-2002, La portée des mots du discours : essai de définition, in Cahiers linguistiques de l'INALCO, ${ }^{\circ} 4$, Paris, Publications Langues'O, p. 930

Culioli A., 1990, Pour une linguistique de l'énonciation. Opérations et représentations, Paris, Ophrys, t.1, p. 135-155.

Garde P., 1963, L'emploi du conditionnel et de la particule by en russe, Aix-enProvence, éd. Ophrys, Publications des annales de la Faculté des Lettres.

Gébert L., 1989, La particule russe by : un problème d'ordre des éléments dans la phrase, Etudes de linguistique, La Licorne, $\mathrm{n}^{\circ} 15$, Poitiers, p. 301-307.

Kor Chahine I., 2001, Esli et l'expression de la condition en russe moderne, Thèse de Doctorat, Université de Provence, Aix-en-Provence

L'Hermitte R., 1982, L'expression de l'hypothèse en vieux-slave et en vieuxrusse, L'information grammaticale, $\mathrm{n}^{\circ} 13$, Paris, p. 15-21

Popova-Bottineau T., 2009, Problema razmeščenija časticy bylo s točki zrenija kommunikativnogo analiza (Le choix du point d'incidence de la particule bylo du point de vue de l'analyse communicative), Voprosy jazykoznanija, n 4 , Moscou, p. $72-86$.

Švedova N. JU., 2005, La langue russe (Russkij jazyk), Moscou, Jazyki slavjanskoj kul'tury.

Unbegaun B ., 1951, Grammaire russe, Lyon, éd. IAC, Collection « Les langues du Monde », Série Grammaire, Philologie, Littérature, vol. V. 